\title{
Raynaud's syndrome and carpal tunnel syndrome
}

\author{
Derek G. Waller ${ }^{1}$ and J. Rodney Dathan ${ }^{2}$
}

${ }^{\prime}$ University of Southampton, Southampton General Hospital; ${ }^{2}$ Royal South Hants Hospital, Southampton S09 4PE, UK.

\begin{abstract}
Summary: We report three cases of Raynaud's syndrome with digital ischaemic ulceration, in association with carpal tunnel syndrome. In all cases, the aetiology of the Raynaud's syndrome was probably unrelated to the nerve compression. However, symptoms were worse on the side of the median nerve lesion in two patients and worse on the side with the most severe nerve dysfunction in the third; symptoms were relieved by carpal tunnel decompression in two patients. We suggest that carpal tunnel syndrome may exacerbate Raynaud's syndrome and should be considered particularly in patients with asymmetrical digital lesions.
\end{abstract}

\section{Introduction}

Raynaud's syndrome has many secondary causes (Blunt \& Porter, 1981) and in many cases is ameliorated by successful treatment of the underlying disorder. The association between carpal tunnel syndrome and digital vasospasm was first recorded by Garland et al. (1957) although the causal relationship has been questioned (Blunt \& Porter, 1981). We describe three cases of Raynaud's syndrome in which the digital ischaemia appears to have been exacerbated by carpal tunnel syndrome.

\section{Case reports}

\section{Case 1}

A male aged 60 presented in 1959 with evidence of a right median nerve lesion. He was subsequently lost to follow-up until 1978 when he developed superficial ulceration of the right index and middle fingers and intermittent pallor of the fingers of that hand. Full blood count was normal and the ESR $53 \mathrm{~mm} / \mathrm{h}$; rheumatoid factor and antinuclear factor were negative. IgG and IgA concentrations were slightly increased but no paraprotein, cryoglobulins, cold agglutinins or immune complexes were detected. An electromyogram (EMG) was consistent with a severe carpal tunnel syndrome on the right.

Derek G. Waller, B.Sc., M.R.C.P.; J. Rodney Dathan M.D., F.R.C.P.

Correspondence: D.G. Waller, Clinical Pharmacology Group, Level F, Centre Block, Southampton General Hospital, Southampton S09 4XY, UK.

Accepted: 22 December 1983
In 1979 he developed symptoms of polymyalgia and treatment was commenced with steroids, with a rapid improvement in the digital signs and symptoms. Carpal tunnel decompression was undertaken and the steroids were discontinued one month later without further digital ischaemia. Six weeks after stopping steroids, the ESR had risen to $108 \mathrm{~mm} / \mathrm{h}$ and they were recommenced.

\section{Case 2}

A male aged 73 presented with a 1 y history of cold fingers, worse on the right. Splinter haemorrhages and ischaemic ulceration were present on all finger pulps, but also worse on the right hand. There was clinical evidence of a right median nerve lesion. A full blood count was normal and the ESR was $6 \mathrm{~mm} / \mathrm{h}$. Rheumatoid factor was negative, antinuclear factor (ANF) was positive but DNA binding was normal. Immunoglobulins were normal and there were no cold agglutinins, but low levels of cryoglobulins and immune complexes were present. An EMG was consistent with a carpal tunnel syndrome on the right. Carpal tunnel decompression was undertaken with considerable improvement in symptoms.

Case 3

A female aged 81 presented with malaise, pyrexia and intermittent pallor of the fingers. There was ischaemic ulceration of the thumb, index and middle fingers of the left hand, with signs of a bilateral median nerve lesion. Full blood count was normal; the ESR was $127 \mathrm{~mm} / \mathrm{h}$. Rheumatoid factor and ANF were negative, immunoglobulins were normal but

(C) The Fellowship of Postgraduate Medicine, 1985 
cryoglobulins and immune complexes were detected. An EMG confirmed bilateral carpal tunnel syndrome, worse on the left. Steroids resulted in rapid resolution of the digital signs. Further investigation revealed the presence of a hepatoma from which she later died.

\section{Discussion}

In all 3 patients, the investigations suggested that the Raynaud's syndrome was due to an arteritis associated with a systemic inflammatory disorder. The signs and symptoms of digital ischaemia were most marked on the side of the carpal tunnel syndrome in cases 1 and 2, and more marked on the side with the more severe median nerve compression in case 3 . None of the patients had clinical evidence of arthritis or synovial thickening affecting the wrist joints.

The association between carpal tunnel syndrome

\section{References}

BLUNT, R.J. \& PORTER, J.M. (1981). Raynaud syndrome. Seminars in Arthritis and Rheumatism. 10, 282.

GARLAND, H., BRADSHAW, J.P.P. \& CLARK, J.M.P. (1957). Compression of median nerve in carpal tunnel and its relation to acroparasthesiae. British Medical Journal, 1, 730. and vasospasm was studied by Linscheid et al. (1967) who demonstrated co-existence of the conditions in $1 \%$ of his 2,800 patients with median nerve lesions. Fourteen of the 28 patients probably had underlying systemic disease, most commonly rheumatoid arthritis and systemic sclerosis. The authors suggested that compression of sympathetic fibres in the median nerve was responsible for vasospasm, but found that surgery was effective in relieving vascular signs and symptoms in only 6 of 24 hands.

In our 3 patients there was an association between the presence and severity of carpal tunnel syndrome and digital ischaemia and cases 1 and 2 responded favourably to surgery. We suggest, therefore, that in patients with Raynaud's syndrome and asymmetry of the severity of symptoms and signs, that evidence of a median nerve lesion should be sought, and surgical decompression considered.

LINSCHEID, R.L., PETERSON, L.F.A. \& JUERGENS, J.L. (1967). Carpal tunnel syndrome associated with vasospasm. Journal of Bone and Joint Surgery, 49A, 1141. 